\title{
Anomalias venosas nos cavernomas
}

\author{
Williams Escalante1, Diana Lara Pinto de Santana, Eberval Gadelha Figueiredo², \\ José Guilherme P. Caldas ${ }^{2}$, Manoel Jacobsen Teixeira ${ }^{3}$ \\ Divisão de Clínica Neurocirúrgica da Faculdade de Medicina da Universidade de São Paulo (FMUSP), São Paulo, SP, Brasil.
}

\section{RESUMO}

As anomalias do desenvolvimento venoso (DVA) são as malformações vasculares cerebrais mais frequentes, tendo geralmente um curso benigno, e podem estar acompanhando os cavernomas. Atualmente, são mais diagnosticadas por causa dos métodos de diagnósticos por imagem que existem, como a tomografia computadorizada e, sobretudo, a ressonância magnética nuclear. Este artigo revisa as DAV em geral e as relacionadas com os cavernomas. Os artigos foram buscados na base de dados PubMed, em publicações desde 1995 a junho de 2012, usando como filtros as expressões "anomalias do desenvolvimento venoso e malformações cavernosas" e "anomalias venosas cerebrais e malformações cavernosas"; a pesquisa foi limitada a artigos em idioma inglês e relacionados a humanos. As sessões de referência de artigos e revisões recentes foram revisadas e os artigos pertinentes foram recuperados no formato resumo; os manuscritos em texto completo foram obtidos subsequentemente para todos os artigos originais aplicados à revisão em curso.

\section{PALAVRAS-CHAVE}

Malformações arteriovenosas intracranianas, ressonância magnética, hemangioma cavernoso.

\section{ABSTRACT}

Venous anomalies in cavernomas

Developmental venous anomalies (DAV) are the most common brain vascular malformations usually, having a benign course and may be following the cavernomas. They are more diagnosed recently due to diagnostic imaging methods that exist, such as computed tomography and nuclear magnetic resonance. This article reviews the DAV in general and those relating to cavernomas. The PubMed database was searched for publications from 1995 through June 2012 using the mesh terms "anomalies venous cerebral, development venous and cavernous malformation", "anomaly venous cerebral and cavernous malformation". The search was limited to articles in the English language and related to humans subjects. Reference sections of recent articles and reviews were reviewed and pertinent articles were retrieved in the abstract format, full text manuscripts were subsequently obtained for all original articles applicable to the current review.

\section{KEYWORDS}

Intracranial arteriovenous malformations, magnetic resonance imaging, hemangioma cavernous.

1 Médico-residente de Neurocirurgia da Faculdade de Medicina da Universidade de São Paulo (FMUSP), São Paulo, SP, Brasil

2 Professor livre-docente da Faculdade de Medicina da Universidade de São Paulo, supervisor da Divisão de Clínica Neurocirúrgica do Hospital das Clínicas da FMUSP, São Paulo, SP, Brasil.

3 Professor titular da disciplina de Neurocirurgia do Departamento de Neurologia da FMUSP, São Paulo, SP, Brasil. 


\section{Introdução}

O termo "anomalia do desenvolvimento venoso (DVA)" é também chamado de angioma venoso. A DVA é uma formação radial de veias que normalmente terminam numa veia central ou principal geralmente dilatada.

A DVA pode drenar tanto no sistema venoso superficial como no profundo, sendo um deles o predominante. O papel essencial desempenhado por uma DVA na drenagem venosa evidencia-se nas complicações da extirpação cirúrgica de uma DVA, resultando em isquemia e hemorragias nas regiões circundantes. ${ }^{1}$

As malformações cavernosas, também chamadas de cavernomas, hemangiomas cavernosos ou angiomas, são malformações estritamente venosas, formadas por canais vasculares muito dilatados e de paredes extremamente delgadas. Na maioria, são constituídas apenas por uma fina camada de fibras colágenas revestidas por células endoteliais. Não há fibras musculares lisas nem lâmina elástica. Algumas paredes mais espessas podem resultar da organização de trombos. Não apresentam tecido neural interposto, grandes artérias aferentes nem veias. ${ }^{2}$

A DVA é a malformação vascular cerebral mais frequente, com uma incidência de até $2,6 \%$ numa série de 4,069 autópsias cerebrais. ${ }^{1}$

Existia controvérsia sobre a origem e patogênese das DVA, que podem ser solitárias ou múltiplas, mas concretamente podem ocorrer em $25 \%$ das malformações cavernosas cerebrais (CCM), pelo que alguns autores sugeriam uma origem comum, porém os estudos genéticos apoiam a hipótese de que DVA e CCM são duas entidades distintas com biologia, mecanismos patogênicos, comportamento clínico e tratamento diferentes. ${ }^{3,4}$

\section{Patologia}

A possibilidade de um mecanismo patológico relacionado a DVA levando ao desenvolvimento de um CCM foi proposta por alguns autores. Foi levantada a hipótese de que a elevada pressão venosa no território de uma DVA daria lugar a uma cascata de eventos que conduziriam à formação do CCM e que estes pareciam surgir nos ramos distais das malformações venosas. Medições elevadas da pressão dentro das DVA sugerem que isso pode ser um fator-chave no desenvolvimento posterior de uma lesão similar ao cavernoma. ${ }^{5}$

A radioterapia também produz hialinização e necrose fibrinoide das pequenas artérias e arteríolas, bem como o estreitamento do lúmen e a proliferação endotelial, produzindo lesões tardias. A radioterapia pode induzir a formação de telangiectasia capilar mediante alterações do endotélio das veias. Todas essas alterações são mais dramáticas nas veias, com dilatação e oclusão das suas paredes, sendo uma causa provável de formação dos cavernomas. ${ }^{6}$

Diante desses fatos, é possível que os cavernomas em associação com as DVA tenham uma origem adquirida em detrimento de uma origem congênita. Outro fato que corrobora essa hipótese é o fato de que a coexistência das malformações cavernosas e as DVA é mais comum nos adultos do que nas crianças. ${ }^{5}$

\section{Diagnóstico clínico}

Geralmente as DVA são assintomáticas, porém quando apresentam sintomas, os mais comuns são cefaleia, crise convulsiva, déficits focais, vertigem e zumbido. Elas também podem ser encontradas acidentalmente na investigação de outras patologias neurocirúrgicas. ${ }^{7}$

\section{Diagnóstico por imagem}

As anomalias do desenvolvimento venoso se apresentam na angiografia com opacificação no tempo normal de enchimento venoso e, em algumas ocasiões, quando localizadas na região frontal, podem ser demonstradas de forma precoce e podem ter associado um blush capilar. Além disso, apresentam pequenas veias agrupadas radialmente, de drenagem profunda, que convergem para uma veia de drenagem mais calibrosa transcortical ou subependimária, com a forma característica de cabeça de medusa. ${ }^{8}$ Os cavernomas não são vistos no exame de arteriografia. Já no exame de ressonância nuclear magnética, as DVA geralmente são vistas na sequência sem contraste como pontos ou linhas de flow-void. Após injeção de contraste há aumento da captação, seguindo um padrão semelhante ao da angiografia. Os cavernomas que podem estar associados às DVA se apresentam como lesões discretas, bem circunscritas, multilobuladas, com conteúdo sanguíneo em diversas fases da degradação, com aspecto definido como semelhante ao da pipoca (Figura 1$)^{9}$.

\section{Tratamento}

Geralmente, o tratamento é expectante, e somente se ocorrerem convulsões que não cedem à terapia farmacológica ou em casos raros e controversos de 
A
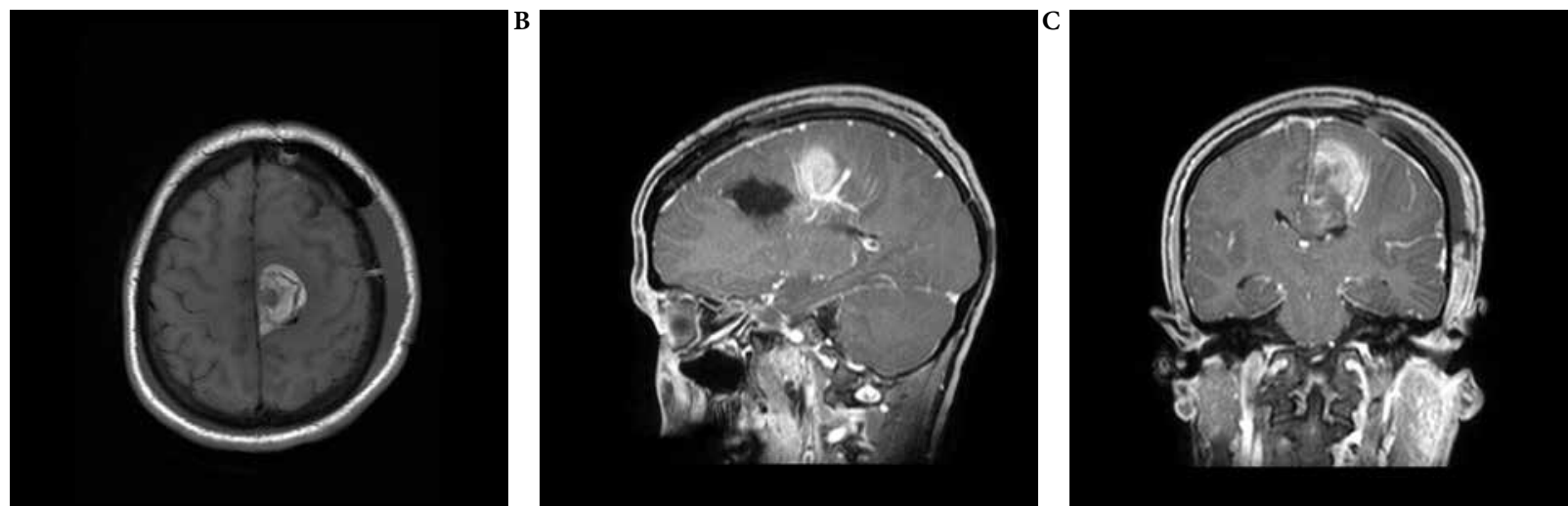

Figura 1 - Imagens no T1 com gadolínio que mostram vasos sanguíneos confluentes do angioma venoso com cavernoma associado, evidenciando-se um vaso dilatado de drenagem e hematoma parassagital esquerdo, na região frontoparietal. (A) Corte axial; (B) Corte sagital; (C) Corte coronal de RMN.

hemorragias associadas estaria indicado o tratamento cirúrgico. Na cirurgia, deve-se preservar a veia principal da DVA durante a exérese cirúrgica de um hematoma ou resseção de um cavernoma situado próximo a ela. ${ }^{1}$

Deve-se evitar ressecar a veia de drenagem da DVA para evitar o risco de infarto venoso. Caso isso ocorra, pode haver consequentemente congestão e edema cerebral, com consequências devastadoras.

Em um relatório recente, foram propostas a coagulação e a divisão da veia da DVA para evitar a recorrência do CCM. Em sua série de 15 pacientes com DVA e CCM, foi realizada essa técnica em nove pacientes; em seis pacientes durante a primeira operação e nos outros três pacientes após recidiva do CCM associado à hemorragia sintomática. Nesses nove pacientes não houve complicações. ${ }^{8,10}$

\section{Referências}

1. Ruíz DS, Yilmaz H, Gailloud P. Cerebral developmental venous anomalies: current concepts. Ann Neurol. 2009;66(3):271-83.

2. Abla A, Wait SD, Uschold T, Lekovic GP, Spetzler RF. Developmental venous anomaly, cavernous malformation, and capillary telangiectasia: spectrum of a single disease. Acta Neurochir (Wien). 2008;150(5):487-9.
3. Guclu B, Ozturk AK, Pricola KL, Seker A, Ozek M, Gunel $M$. Cerebral venous malformations have distinct genetic origin from cerebral cavernous malformations. Stroke. 2005;36(11):2479-80.

4. Dillon WP. Cryptic vascular malformations: controversies in terminology, diagnosis, pathophysiology, and treatment. AJNR Am J Neuroradiol. 1997;18(10):1839-46.

5. Campeau NG, Lane JI. De novo development of a lesion with the appearance of a cavernous malformation adjacent to an existing developmental venous anomaly. AJNR Am J Neuroradiol. 2005;26(1):156-9.

6. Maeder P, Gudinchet F, Meuli R, De Tribolet N. Development of a cavernous malformation of the brain. AJNR Am J Neuroradiol. 1998;19(6):1141-3.

7. Töpper R, Jürgens E, Reul J, Thron A. Clinical significance of intracranial developmental venous anomalies. J Neurol Neurosurg Psychiatry. 1999;67(2):234-8.

8. Hon J, Bhattacharya J, Counsell C, Papanastassiou $\mathrm{V}$, Ritchie V, Roberts RC, et al. The presentation and clinical course of intracranial developmental venous anomalies in adults. Stroke. 2009;40:1980-5.

9. Obstertun B, Solymosi L. Magnetic resonance angiography of cerebral developmental venous anomalies: its role in differential diagnosis. Neuroradiology. 1993;35:97-104.

10. Wurm G, Schnizer M, Fellner FA. Cerebral cavernous malformations associated with venous anomalies: surgical considerations. Neurosurgery. 2005;57(Suppl 1):42-58.

\section{Endereço para correspondência}

Diana Lara Pinto de Santana

Av. Dr. Enéas de Carvalho Aguiar, 255, Cerqueira César

05403-000 - São Paulo, SP, Brasil

Telefone: (+55 11) 99733-0208

E-mail: didisantana@yahoo.com.br 